\title{
Radio emissions from terrestrial planets around white dwarfs
}

\author{
A. J. Willes ${ }^{1}$ and $\mathrm{K} \cdot \mathrm{Wu}^{2}$ \\ 1 British Antarctic Survey, Cambridge, UK and School of Physics, University of Sydney, NSW 2006, Australia \\ e-mail: A.Willes@physics.usyd.edu.au \\ 2 Mullard Space Science Laboratory, University College London, Holmbury St. Mary, Surrey, UK \\ e-mail: kw@mssl.ucl.ac.uk
}

Received 10 March 2004 / Accepted 19 November 2004

\begin{abstract}
Terrestrial planets in close orbits around magnetic white dwarf stars are potential electron-cyclotron maser sources, by analogy to planetary radio emissions generated from the electrodynamic interaction between Jupiter and the Galilean moons. We present predictions of radio flux densities and the number of detectable white-dwarf/terrestrial-planet systems, and discuss a scenario for their formation.
\end{abstract}

Key words. stars: planetary systems - stars: white dwarfs - radiation mechanisms: non-thermal - radio continuum: general

\section{Introduction}

In the unipolar inductor model for the Jupiter-Io electrodynamic interaction (Piddington \& Drake 1968; Goldreich \& Lynden-Bell 1969) a current circuit is set up along the magnetic field lines connecting Io to Jupiter. The currents are driven by the potential induced across Io, a conducting body, as it traverses the jovian magnetic field. The high energy electrons carrying the current produce an auroral footprint in Jupiter's atmosphere (Clarke et al. 2002). The energetic electrons also produce radio emissions, via the electron cyclotron maser mechanism, which generates a "hollow-cone" radiation pattern (Hewitt et al. 1981). The predicted beaming is consistent with ground and multi-spacecraft observations, which also locate the source near the base of the Io flux tube (Bigg 1964; Dulk 1967; Maeda \& Carr 1992; Kaiser et al. 2000).

The electrodynamics in the Jovian system is complicated by the presence of a dense plasma torus surrounding Io's orbit, which inhibits the rapid communication of magnetic stresses (carried by Alfvén waves) between Io and Jupiter's ionosphere. Io's auroral footprint has two components: a bright point source at the base of the instantaneous Io flux tube, consistent with the predictions of the unipolar inductor model, and a long diffuse tail, which signifies an extended field-aligned current circuit associated with the transfer of momentum from the Io plasma torus to the stagnant plasma in Io's wake (Delamere et al. 2003; Hill \& Vasyliunas 2002).

Europa, Ganymede and Callisto also control jovian decametric radiation, although to a lesser degree than Io (Menietti et al. 1998, 2001), and Ganymede and Europa also cast auroral footprints. The unipolar inductor model is also applicable to systems where Jupiter is replaced by a magnetic white dwarf. Fast Alfvén speeds associated with high white dwarf magnetic moments ensure rapid communication of magnetic stresses along the connecting flux tube. The role of the inductor in white dwarf systems can be played by a red dwarf (in AM Her binaries, Chanmugam \& Dulk 1983), a terrestrial planet core (Li et al. 1998), or an unmagnetized white dwarf (Wu et al. 2002). The induced potential and dissipated power in white-dwarf systems is typically significantly higher than in the Jupiter-Io system.

The most compelling evidence for the applicability of the unipolar inductor model to white-dwarf systems comes from timing measurements of optical and X-ray emissions from two close white-dwarf pairs. A unipolar inductor model readily explains the anti-phased X-ray and optical/IR pulses emitted by RX J1914+24 and RX J0806+15 (Wu et al. 2002; Ramsay et al. 2002). The $X$-rays are powered by resistive heating of the magnetic white-dwarf atmosphere at the base of the flux tube, analogous to the ultraviolet auroral footprints of the Galilean moons in Jupiter's atmosphere. A sub-10-min orbital period white dwarf pair can produce a footpoint X-ray luminosity well exceeding the solar bolometric luminosity, with only very modest departures from spin/orbit synchronism. The anti-phased optical/infrared emission emanates from the irradiated face of the nonmagnetic white dwarf, which appears as the X-ray source rotates out of view. There is no mass transfer between the white dwarfs, and the orbital period gradually decreases when gravitational radiation from the binary system carries away the orbital angular momentum. The unipolar inductor model is thus consistent with precise timing measurements of X-ray pulses from RX J1914+24 (Strohmayer 2002), and X-ray and optical pulses from RX J0806+15 (Hakala et al. 2003; Strohmayer 2003). In both cases, the orbital period decreases at a rate consistent with gravitational radiation losses, which contradicts the 


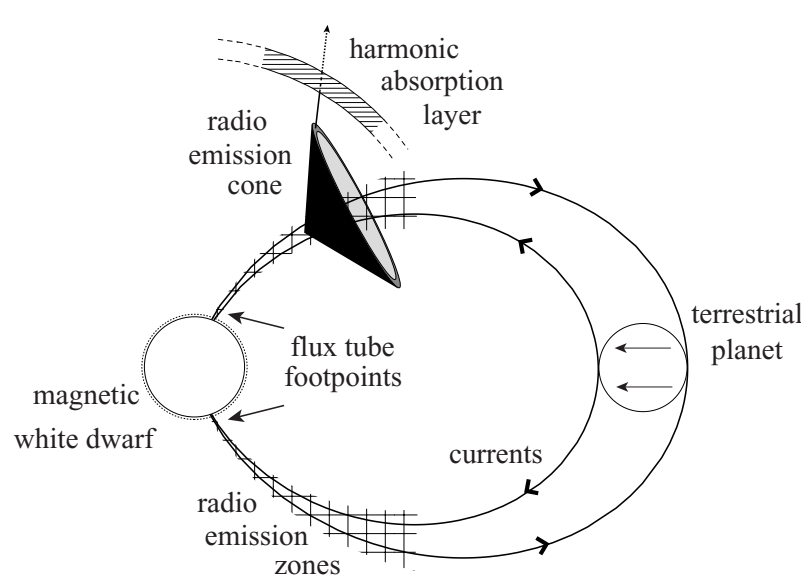

Fig. 1. Unipolar inductor model for white-dwarf systems.

predicted period increase by alternative models, which invoke steady mass transfer between the white dwarfs to explain the $\mathrm{X}$-ray emission.

The unipolar inductor model for terrestrial planets in close orbits around magnetic white dwarfs was originally constructed by $\mathrm{Li}$ et al. (1998) to explain the emission lines detected in the white dwarf system GD 356. Willes \& Wu (2002) proposed that white-dwarf planet systems undergoing the unipolar induction process are also detectable radio sources. More definitive predictions are made in this paper, which is structured as follows. In Sect. 2, we deduce the range of parameters (white dwarf magnetic moment and orbital period) in which detection of white-dwarf/planet systems is most likely. In Sect. 3, we discuss an evolution scenario for close white-dwarf/planet systems. We estimate the fraction of main-sequence-star/terrestrial-planet systems which evolve into white-dwarf/planet systems operating in the unipolar inductor regime. In Sect. 4, we present predictions of radio flux densities from the systems, based on the electron-cyclotron maser model for white dwarf systems developed by Willes \& Wu (2004), at frequencies accessible to the VLA, ALMA, Arecibo, ATCA, MERLIN and Effelsberg radio telescopes. In Sect. 5, we compute the radio luminosity function of the whitedwarf/planet systems and estimate the number of detectable systems for various observational settings.

\section{Detectability of terrestrial planets orbiting magnetic white dwarfs}

Figure 1 shows a schematic illustration of the system geometry. In our model, the system comprises a magnetic white dwarf with mass $M_{\mathrm{WD}}$ and magnetic moment $\mu$ and a terrestrial planet with mass $M_{\mathrm{P}}$. The white dwarf radius $R_{\mathrm{WD}}$ is given by the Hamada \& Salpeter (1961) mass-radius relation. The terrestrial planet is assumed to have a metallic core which is exposed during the late stellar evolution phases. Electron currents are driven in the flux tube current circuit as the planet traverses the magnetic white dwarf's field lines, inducing a potential difference across the planet. The induced currents are carried by mildly relativistic electrons. These energetic ( $>\mathrm{keV})$ electrons

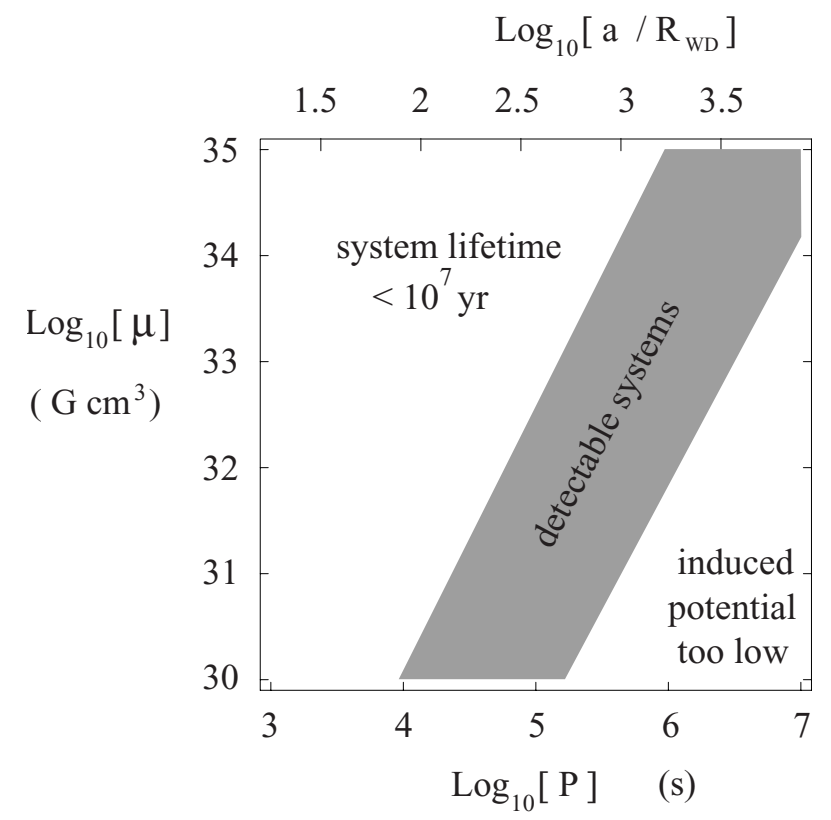

Fig. 2. Range of parameter space (white dwarf magnetic moment $\mu$ vs. orbital period $P$ and separation $a$ ) in which terrestrial planets orbiting white dwarfs are likely to produce detectable radio emissions.

are the source of free energy for wave growth via the electroncyclotron maser.

The induced potential across a terrestrial planet (see Fig. 1) is (Wu et al. 2002)

$\Phi=\left(\frac{\mu R_{\mathrm{P}}}{c}\right)\left(\frac{2 \pi}{P}\right)^{7 / 3}\left[G\left(M_{\mathrm{WD}}+M_{\mathrm{P}}\right)\right]^{-2 / 3}$,

where $\mu$ is the white dwarf magnetic moment, $R_{\mathrm{P}}$ is the planetary radius, $P$ is the orbital period, and $M_{\mathrm{WD}}, M_{\mathrm{P}}$ are the white dwarf and planetary masses. The detectability of a system depends the brightness of its maser emission, which is contingent on the avaliability of energetic electrons. This condition is easily satisifed for systems with reasonable parameter values. Figure 2 shows the region of $\mu-P$ space in which the induced potential exceeds one $\mathrm{kV}$ (which consists of the grey band and the region to its left) for a characteristic white-dwarf planet system with $M_{\mathrm{WD}}=0.7 M_{\odot}, M_{\mathrm{P}}=1.2 \times 10^{-6} M_{\odot}$, and $R_{\mathrm{P}}=3.5 \times 10^{8} \mathrm{~cm}$.

Another constraint to the probability of detection for whitedwarf planets is the lifetime of the unipolar-inductor phase and the system lifetime. The system lifetime is limited by the slow inward drift of the planet due to Lorentz torques ( $\mathrm{Li}$ et al. 1998), with

$\tau=\frac{G M_{\mathrm{WD}} M_{\mathrm{P}}\left(a / R_{\mathrm{WD}}\right)^{1 / 2}}{60 \sigma R_{\mathrm{WD}} R_{\mathrm{P}} \Phi^{2}}$,

where $a$ is the orbital separation. This lifespan thus scales with binary period as $P^{5}$ and with magnetic moment as $\mu^{-2}$. In Fig. 2, we show the region of $\mu-P$ space in which the system lifetime exceeds 10 million years, (which includes the grey band and the region to its right). The grey band thus represents the optimal region in the $\mu-P$ space where a whitedwarf/terrestrial-planet system satisfies the "detectablity" criteria, i.e., it is capable of generating bright radio emissions, 

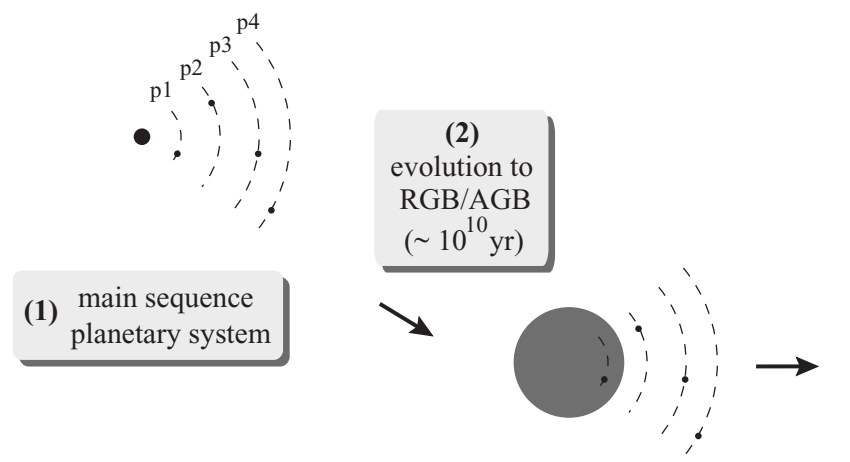

(1)

main sequence planetary system
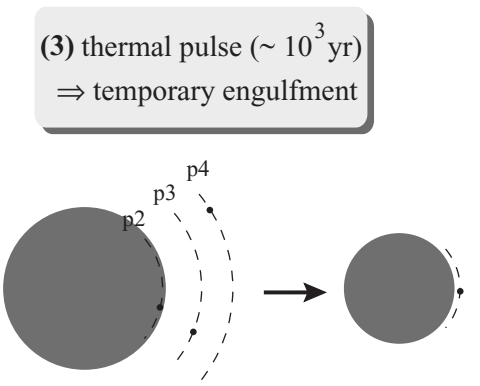

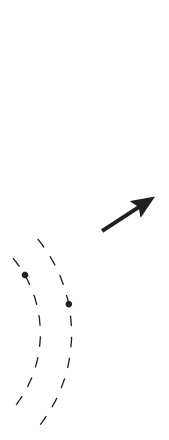

(4) white dwarf

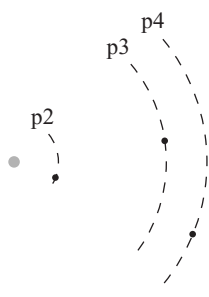
planetary system

Fig. 3. Evolution scenario for white-dwarf/terrestrial-planet systems.

and the lifetime of the radio-emitting phase is not prohibitively short.

\section{Evolution from main sequence to white dwarf planetary systems}

We now examine the question of whether or not whitedwarf/terrestrial-planet systems can be formed. Consider a system consisting of a main-sequence (MS) star with a number of orbiting planets. Like the solar system, the inner planets are assumed to be terrestrial and have a solid core. The star eventually evolves beyond the main sequence and enters a giant phase. If the evolved system is to be in the parameter regime where detectable radio emissions are produced (see Fig. 2), these planets must not only survive the major stellar expansion phases, but also retain a close orbit around the emergent white dwarf. At first sight this appears to be difficult because the stellar envelope would expand beyond expected terrestrial planet orbits (for example, beyond the orbit of Venus in our solar system) during the red giant branch phase (after $\sim 10^{10}$ years, as hydrogen burning in the core ceases). We note as an aside that one interpretation of the unusual light curve of the enigmatic system V838 Monocerotis is the successive engulfment of three close-orbit planets by the expansion of a red giant (Retter \& Maron 2003).

However, the planetary orbits vary substantially during the post-MS stellar evolution. There are a number of competing effects which determine the evolution of the planetary orbits; the four main effects are: (i) Conservation of angular momentum ensures that the orbits expand as the star experiences significant mass loss during the red giant branch (RGB) and asymptotic giant branch (AGB) phases (Sackmann et al. 1993). (ii) Tidal interactions cause a transfer of orbital angular momentum from the planet to spin up the stellar envelope, leading to a decay of the planetary orbit. The orbital decay rate varies as $\left(R_{\text {star }} / a\right)^{8}$, and so dominates over mass-loss effects as the stellar envelope approaches the planet (Rasio et al. 1996; Rybicki \& Denis 2001). (iii) When the planet is engulfed by the stellar envelope, the orbit rapidly decays due to "bow shock" drag. This typically leads to the destruction of the planet in the stellar interior. However, one important exception is during the AGB phase, when post-main-sequence stars often undergo thermal pulses (or helium flashes, associated with helium burning in the shell), and the stellar radius expands by up to $50 \%$ and recontracts over $1000 \mathrm{yr}$ timescales in each pulse. The thermal pulses provide another mechanism (besides tidal drag) to bring terrestrial planets into closer orbits. This is illustrated schematically in Fig. 3, where four terrestrial planets (p1, p2, p3 and p4) orbit a MS star (stage 1). When the star enters the red giant branch phase, the innermost planet $\mathrm{p} 1$ is permanently engulfed (stage 2). At stage 2, planet p2 may move inwards due to tidal drag, and planets $\mathrm{p} 3$ and $\mathrm{p} 4$ move outwards due to stellar mass loss. At stage 3, planet p2 undergoes temporary engulfment, and it is overtaken by the stellar envelope over the thermal pulse timescale. Rybicki \& Denis (2001) estimate that a terrestrial planet orbit will decay by $\sim 10-70 R_{\odot}$ per thermal pulse (we argue below that the orbital decay during each pulse is significantly lower than this estimate). The stellar radius then recontracts back towards its quiescent level and the planet remains in a contracted orbit external to the star. After one or more thermal pulses, the planet $\mathrm{p} 2$ is dragged into a closer orbit through a combination of bow shock and tidal drag forces. (iv) For systems which evolve to a white-dwarf/terrestrial-planet system, the planet's orbit will decay further due to electrical dissipation in the unipolar inductor system ( $\mathrm{Li}$ et al. 1998), as discussed in the previous section.

It is thus conceivable that a terrestrial planet not only survives the RGB and AGB expansion phases (through orbital expansion associated with stellar mass loss), but also that it can be dragged back into a closer orbit around the protowhite dwarf by a combination of tidal drag and temporary engulfment effects. In this evolution scenario only a small fraction of MS-star/terrestrial-planet systems will evolve into white-dwarf/terrestrial-planet systems capable of generating detectable radio emission (i.e., in the parameter range illustrated in Fig. 2).

In this paper, we make an estimate of this fraction by tracing planetary orbits within a stellar evolution model, including mass loss, tidal drag and temporary engulfment effects. The following assumptions are made in this model:

(i) We adopt an existing solar evolution model, which traces the evolution of the stellar mass, the convective envelope mass, and the stellar radius from the MS through the RGB and AGB phases (including thermal pulses) to the protowhite dwarf stage (Sackmann et al. 1993, Table 2, with mass-loss parameter $\eta=0.6$; henceforth referred to as the SBK model). 
(ii) The evolution of the orbital separation of the star and planet as a function of time $a(t)$ is modelled using the equation (Rybicki \& Denis 2001):

$$
\begin{aligned}
\frac{1}{a} \frac{\mathrm{d} a}{\mathrm{~d} t}= & -\frac{1}{M} \frac{\mathrm{d} M}{\mathrm{~d} t}-\frac{12 K_{2} M_{\mathrm{P}}}{\tau_{F} M}\left(\frac{R}{a}\right)^{8} \\
& -H(R-a) \frac{2 \pi \rho v_{\text {orb }} C_{\mathrm{D}} R_{\mathrm{P}}^{2}}{M_{\mathrm{P}}}
\end{aligned}
$$

where $v_{\text {orb }}$ is the planet's (Keplerian) orbital velocity, $C_{\mathrm{D}}$ is the drag coefficient, which is of order unity (we assume $C_{\mathrm{D}}=1$ here), and $\rho$ is the stellar envelope density. The first term on the right-hand side of Eq. (3) represents the mass loss, the second term represents the tidal drag, and the third term represents the bow shock drag, which acts only when the orbital separation is less than the stellar radius (where $H$ is the Heaviside step function). Following Rybicki \& Denis (2001), we assume that the apsidal motion constant $K_{2}$ is proportional to the ratio of the convective envelope mass to the total stellar mass (Soker \& Livio 1994):

$K_{2} \approx 0.34 \frac{M_{\mathrm{env}}}{M}$

and that the eddy viscosity timescale has the value $\tau_{F}=$ $2 \times 10^{7}$ (Verbunt \& Phinney 1995). We neglect the "gravitational" drag, which occurs as an engulfed planet accretes stellar material by gravity. For terrestrial planets, this effect is weak in comparison to the bow shock drag (but becomes dominant for Jupiter-sized gaseous planets; Rybicki \& Denis 2001). We also neglect any mass change of terrestrial planets during the temporary engulfment phases. Thus we are effectively assuming a balance between the mass gained by a planet due to accretion of stellar material and mass loss due to evaporation. This approximation is appropriate in our present application to provide a simple estimate of the fraction of planets which survive the post-MS stellar expansion phases. (A more rigorous treatment is, however, necessary in order to make accurate predictions on the fate of individual planets.)

(iii) We assume that the stellar radius peak during the AGB phase is substantially higher than the peak RGB radius. This is the case, for instance, in stellar evolution calculations over wide ranges of input parameters, where the AGB always extends to higher luminosities and stellar radii than the RGB (Boothroyd \& Sackmann 1988b). Given this assumption, it is likely that that the planetary orbits which survive the AGB and thermal pulse phases are sufficiently distant from the peak RGB radius, such that only mass loss effects are significant on the RGB, and that tidal and engulfment effects do not affect the planetary orbit evolution until the AGB.

(iv) The relative temporal spacing of the luminosity and stellar radius peaks and dips within each thermal pulse cycle are modelled from Fig. 5 in Boothroyd \& Sackmann (1988). (v) The density profile of the stellar convective envelope is assumed to have the following radial dependance:

$$
\begin{aligned}
\log \left[\rho\left(\frac{r}{R_{\text {star }}}\right)\right]= \\
\quad\left\{\begin{array}{l}
-11-20 \log \left[\frac{r}{R_{\text {star }}}\right]-0.1<\log \left[\frac{r}{R_{\text {star }}}\right]<0 \\
-9.1-\log \left[\frac{r}{R_{\text {star }}}\right] \quad-1<\log \left[\frac{r}{R_{\text {star }}}\right]<-0.1
\end{array}\right.
\end{aligned}
$$

based on a model of the density profile in the convective envelope of AGB stars (Fig. 1 of Soker 1992), when the mass in the convective envelope satisfies $M_{\text {env }}<0.01 M_{\odot}$ (this condition is typically satisfied during the thermal pulse phase of stellar evolution). The density profile (5) tends to mildly overestimate the density, and therefore the drag, in comparison to the Soker (1992) model, particularly during the last pulses and collapse to a proto-white dwarf. We note here that the approximate expression for the density profile (Eq. (12) in Soker \& Livio 1994), used in this context by Rybicki \& Denis (2001), overestimates the density in the outer stellar envelope and thus significantly overestimates the amount of bow shock drag experienced by a terrestrial planet during each thermal pulse.

The planetary orbit evolution calculation presented here improves on similar previous calculations in two ways. Firstly, stellar mass loss, tidal drag and bow shock drag effects are all included. In contrast, Sackmann et al. (1993) neglect both the tidal and bow shock drags, and Rybicki \& Denis (2001) do not include tidal drag during the AGB and thermal pulse phases (in their Fig. 1). The second improvement over previous calculations is that the variation of the apsidal motion constant $\left(K_{2}\right)$ with the variation in the ratio of envelope to stellar mass is included. This is significant because the degree of tidal drag decreases significantly in the late stages of thermal pulsing in the asymptotic giant branch phase and during the contraction of the star to a proto-white dwarf, as a consequence of the relatively low envelope mass.

Figure 4 illustrates how the competing effects (stellar mass loss, tidal drag, and bow shock drag) influence the evolution of one particular terrestrial planetary orbit (assuming an initial orbital separation of $a_{0}=121.9 R_{\odot}$, and the SBK solar evolution model). The orbital separation initially increases in accordance with stellar mass loss during the RGB and AGB phases. During the AGB phase tidal drag dominates over mass loss effects as the stellar radius approaches the planetary orbit. The combination of tidal and bow shock drag then brings the planet into a closer orbit. The strength of the tidal drag force weakens with each successive thermal pulse in association with a decreasing envelope to total stellar mass ratio. During each temporary engulfment the orbital separation is reduced by $<5 R_{\odot}$. Note that this is an order of magnitude lower than the (10-70) $R_{\odot}$ decay per pulse estimated by Rybicki \& Denis (2001), which results from their overestimate of the gas density in the outer convective envelope of the star. We also note from Fig. 4 that the planet can also survive long engulfments during the interpulse phase, provided that it doesn't stray too deep within the convective stellar envelope, where the higher drag rate 


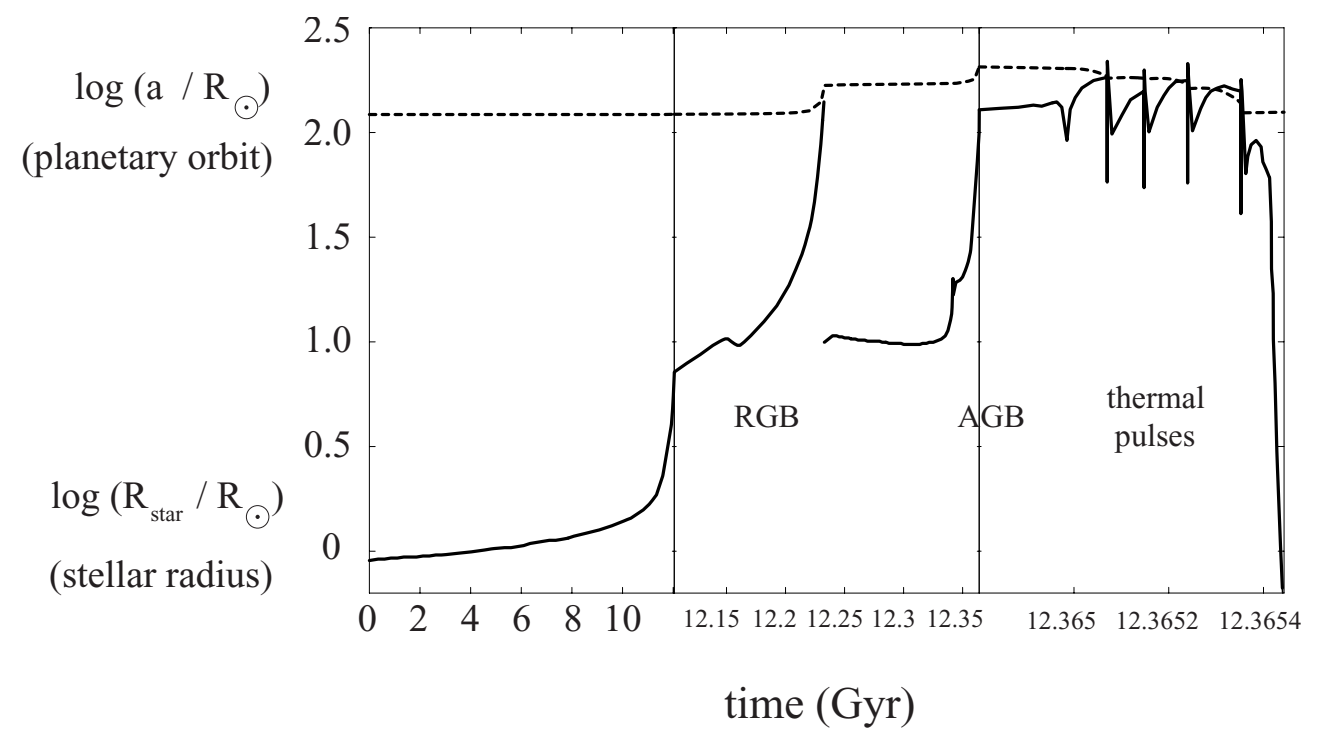

Fig. 4. Evolution of stellar radius [solid line; from Sackmann et al. (1993) SBK model] and planetary orbit (dashed line) for initial orbital separation $a_{0} / R_{\odot}=121.9$. The time axis is separated into three regions, with increasing time resolution. The planet is assumed to have an Earth mass and radius.

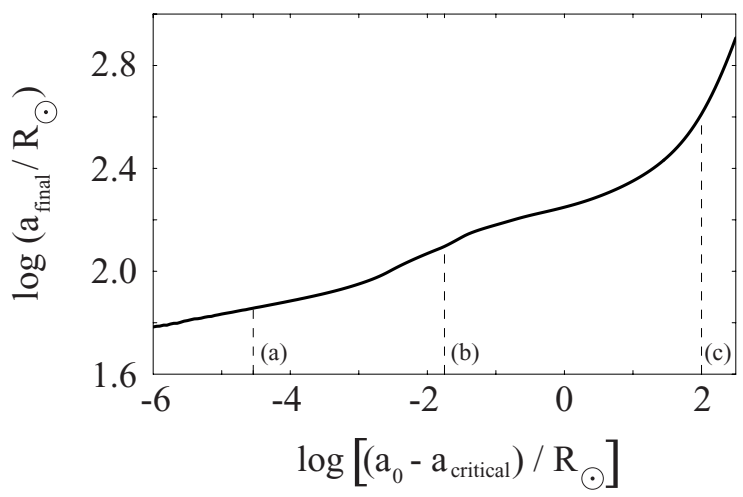

Fig. 5. Final (post-AGB) orbital separation $a_{\text {final }}$ as a function of initial orbital separation $a_{0}$, for initial separations exceeding the critical value $a_{\text {critical }} / R_{\odot} \sim 121.88$. The evolution of the orbital separation at points (a), (b) and (c) (marked by dashed lines) are displayed in Fig. 6.

associated with the increasing gas density causes the planet to rapidly spiral in towards the stellar core. For initial orbital separations less than $a_{\text {critical }} \sim 121.88 R_{\odot}$ (assuming model parameters as in Fig. 4) the planet is permanently engulfed.

Figure 5 plots the final orbital separation $a_{\text {final }}$ (after the star has evolved to a proto-white dwarf) as a function of the initial orbital separation $a_{0}$ (above the critical value $a_{\text {critical }}$ ). Figure 6 traces the orbital separation and stellar radius during the AGB phase for three values of $a_{0}$ (marked by dashed lines in Fig. 5). In panels (a) and (b) tidal and bow shock drag dominate over orbital expansion due to stellar mass loss, and the orbital separation decreases. In panel (c) the initial orbital separation exceeds the maximum stellar radius during the thermal pulse phase and the orbit expands in association with stellar mass loss.

From Fig. 5, the range of initial orbital separations which map to a final orbital separation within $1 \mathrm{AU}\left(215 R_{\odot}\right)$ is $0 \leq a_{0}-a_{\text {critical }} \leq 7 R_{\odot}$, and to inside the orbit of Venus $\left(\sim 150 R_{\odot}\right.$, corresponding to $\log _{10}\left[a / R_{\mathrm{WD}}\right]<4.2$ in Fig. 2 ,
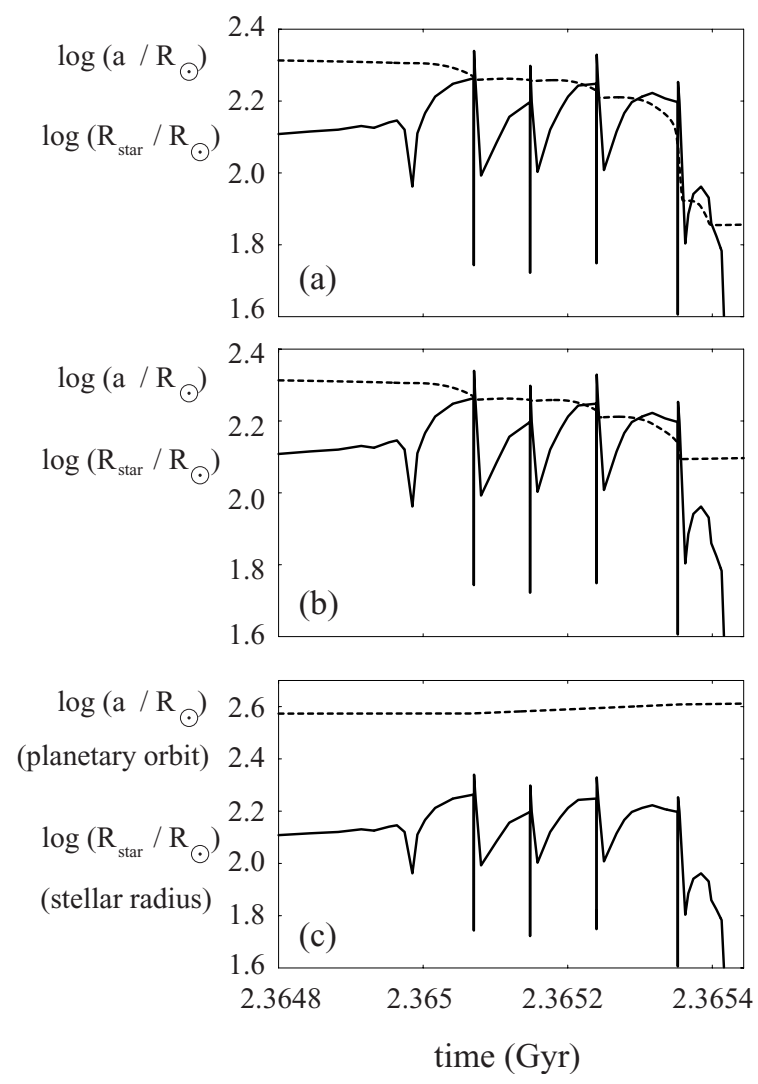

Fig. 6. Evolution of planetary orbits during the asymptotic giant branch and thermal pulse phases for an initial orbital separation: a) $a_{0} / R_{\odot}=121.8822$, b) $a_{0} / R_{\odot}=121.9$, and c) $a_{0} / R_{\odot}=220$.

within the parameter range of observable radio-emitting white$\mathrm{dwarf} /$ planet systems) is $0 \leq a_{0}-a_{\text {critical }} \leq 0.13 R_{\odot}$. If we assume that each MS stellar system has 4 terrestrial planets with initial orbital separations $a_{0} \leq 500 R_{\odot}$, then the fraction 
of systems $\Phi$ that evolve to a WD/planet system in the unipolar inductor regime (with $a_{\mathrm{final}}<150 R_{\odot}$ ) is $\Phi=10^{-3}$ (or $0.1 \%$ ).

This fraction will be lower if not all MS stars host terrestrial planets. However there is a reasonable likelihood that the fraction of MS stars with terrestrial planets is high, based on: (i) Planetary formation simulations, which suggest that terrestrial planets are a common byproduct of star formation and the number and radial distribution of terrestrial planets are not strongly dependent on the stellar mass (Wetherill 1996). In addition, in stellar systems with Jupiter-mass planets in close orbits $(<1 \mathrm{AU})$, a significant fraction of terrestrial planets can survive the inwards orbital migration of Jupiter-mass planets from their formation site at large orbital separations (Mandell \& Sigurdsson 2003). (ii) One explanation for the high incidence of moderately elliptical planetary nebulae is that $\sim 50 \%$ of planetary nebula progenitors host close, low-mass planets (as low as $\sim 0.01 M_{\mathrm{J}}$; Soker 2001).

This calculation demonstrates that the number of observable magnetic white-dwarf/terrestrial planet systems is not prohibitively small. We now discuss how this estimate for $\Phi$ changes with variations in the stellar mass, metallicity and mass-loss rate. The SBK stellar evolution predictions are strongly dependent on the stellar mass-loss rate (where the above estimate is based on the SBK preferred mass-loss parameter of $\eta=0.6$ ). For a lower mass-loss rate (with $\eta=0.4$ ) the star loses less mass on the red giant branch, and thus stays longer on the asymptotic branch, requiring a larger number of thermal pulses to eject the envelope mass (with ten thermal pulses predicted instead of four). There is a corresponding increase in the decay of the planetary orbits because: (i) the expansion of planetary orbits due to stellar mass loss is weaker; (ii) the tidal drag occurs over a longer timescale (by a factor of two); and (iii) the planet encounters a larger number of thermal pulses (however, this is not as strong an effect because each thermal pulse entails an inward drift of only a few $R_{\odot}$ ). Consequently, in the low mass-loss case, it is more likely that close-in planets will be permanently engulfed. However, this will be roughly balanced by more distant planets being dragged into the temporary engulfment regime. Thus, the effect of doubling both the AGB timescale and the number of thermal pulses is that $a_{\text {critical }}$ increases, but the curve in Fig. 5 (and likewise, the estimated value of $\Phi$ ) remains approximately unchanged. This argument, however, does not take into account the fact that variations in the stellar mass-loss rate lead to variations in the predicted (quiescent or inter-pulse) stellar radius on the AGB, and that this has a significant impact on the estimated fraction of close planets $\Phi$. For example, from a comparison of SBK Figs. 7a and 7b, the peak quiescent AGB radius is approximately $25 \%$ higher in the low mass-loss case. The curve in Fig. 5 is shifted upwards by a corresponding amount, which has the effect of reducing $\Phi$ by approximately an order of magnitude. Similarly, by increasing the mass-loss rate (from $\eta=0.6$ ), the peak inter-pulse AGB radius will decrease, where a decrease in the peak inter-pulse AGB radius by $\sim 25 \%$ will increase $\Phi$ by an order of magnitude to $\sim 1 \%$. We note that other solar evolution models yield a wide range of predictions for the peak RGB and AGB solar radii, depending on the choice of mass-loss model, the mass-loss parameter, and the initial chemical composition (e.g., Jørgensen 1991). For example, in the penultimate model in Table 1 of Jørgensen (1991), the (quiescent) AGB peak occurs at $0.74 \mathrm{AU}$, as compared with 0.84 $\mathrm{AU}$ in the SBK model. In addition, there is a large uncertainty in the convection parameter $\alpha$ assumed in the mixing length theory used to model stellar convection (Boothroyd \& Sackmann 1988b). Variation of $\alpha$ within the range of uncertainty has a large effect on the predicted stellar radius and mass loss rate. Sackmann et al. (1993) also discuss a high mass-loss case (with $\eta=1.4$ ). In this extreme example the star loses considerably more mass as it approaches the tip of the RGB, and it bypasses the AGB and thermal pulse phases and directly evolves to the planetary nebula and white dwarf phases. In this case the estimated fraction of close planets is likely to be considerably lower.

Apart from the mass-loss rate, the evolution of intermediate mass stars is principally dependent on the core mass and metallicity (Boothroyd \& Sackmann 1988a). Two stars with the same core mass and metallicity but different total masses evolve similarly, except that the higher-mass star remains longer on the AGB and emits more thermal pulses to expel its envelope. Boothroyd \& Sackmann (1988a) studied the stellar evolution of stars with low metallicity $(Z=0.001)$ and solar metallicity $(Z=0.02)$, finding that stars with mass $<1.2 M_{\odot}$ exhibit fewer than 7 thermal pulses. This increases to 21-22 pulses for a $3 M_{\odot}$ solar-metallicity star. This suggests that the adopted SBK model (with 4 thermal pulses) is representative of a wide range of metallicities, and stellar masses $<1.2 M_{\odot}$. Further calculations are necessary to estimate $\Phi$ for higher-mass stars with many thermal pulses (for $M>2 M_{\odot}$ ). The most significant effect of increasing the initial total stellar mass is that it yields a higher stellar radius in the AGB phase, with a corresponding decrease in the estimated fraction of close planets (as discussed above). For example, increasing the initial mass to $1.2 M_{\odot}$ leads to an increase in the quiescent AGB radius of $\sim 25 \%$ (with an associated order of magnitude decrease in $\Phi)$. Correspondingly, systems with initial stellar masses below $1 M_{\odot}$ will produce more close planets. Variations in the stellar metallicity only weakly affect predictions of the AGB stellar radius (Boothroyd \& Sackmann 1988a), and thus should not significantly affect the above estimate for $\Phi$.

\section{Radio flux density predictions}

In this section we briefly outline the model used to predict the radio flux densities from white dwarf systems with an emphasis on defining free parameters in the model. The complete model is detailed in Willes \& Wu (2004). The convergence of the flux tube magnetic field lines near the magnetic white dwarf surface provides the ideal geometry for a loss-cone instability to form in the mildly relativistic electron velocity distribution. The loss cone is produced by the preferential magnetic mirroring of electrons with large pitch angles. The loss-cone electron density parameter is defined as the loss-cone number density at the mirror point at the base of the flux tube. The electron number density decreases with distance along the flux tube, as the flux tube radius increases, such that the integrated electron density across the flux tube cross section remains 

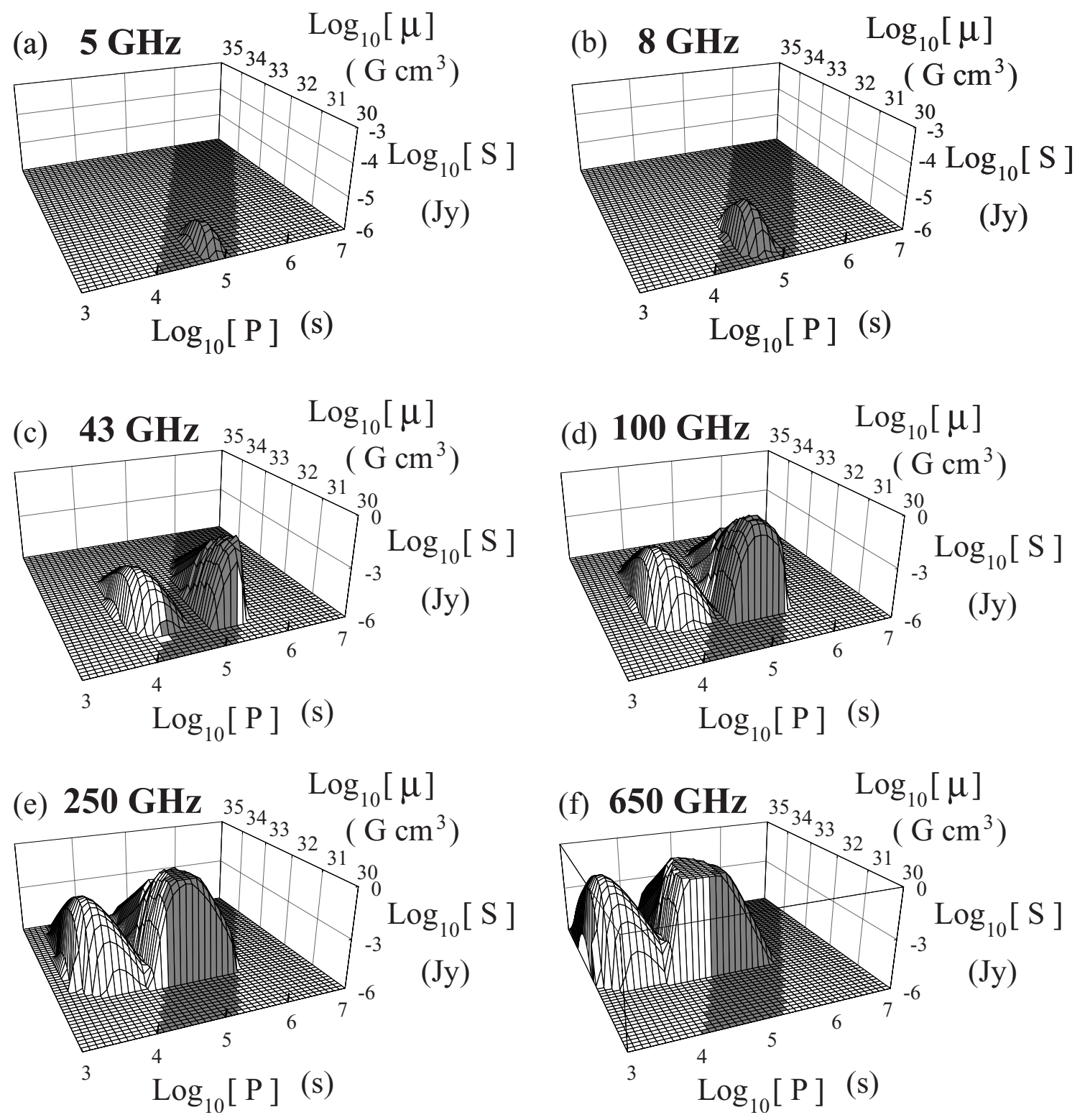

Fig. 7. Predicted flux densities at 5, 8, 43,100, 250 and $650 \mathrm{GHz}$ as a function of white dwarf magnetic moment $\mu$ and planetary orbital period $P$ (assuming system parameters described in the text). The grey band denotes the detection band in Fig. 2.

constant. Willes \& Wu (2004) derived an expression for radio flux densities generated within the white dwarf pair unipolar inductor circuit, based on semi-quantitative expressions for the loss-cone-driven electron-cyclotron maser growth rate, emission bandwidth, and angular ranges, obtained by Melrose \& Dulk (1982). The highest maser growth rates are attained in the extraordinary $(x)$ and ordinary $(o)$ modes at the electron cyclotron frequency. The electron-cyclotron maser thus produces $100 \%$ circularly polarized emission. Higher cyclotron harmonics have considerably lower growth rates and are unlikely to be observed. The peak brightness temperature in the source region is estimated by assuming saturation of the reactive version of the electron-cyclotron maser instability at wave levels where electrons become trapped in the electric field of the growing waves. Maser radiation encounters harmonic damping as it propagates away from the source region towards decreasing magnetic field strengths. The degree of harmonic damping depends on the thermal (background) electron density $n_{\text {th }}$, and temperature $k_{\mathrm{B}} T_{\mathrm{th}}$. Harmonic damping effects become negligible for $n_{\text {th }}<10^{8} \mathrm{~cm}^{-3}$ and $k_{\mathrm{B}} T_{\text {th }}<10 \mathrm{eV}$ (Willes \& Wu 2004).

In the following flux density calculations, we assume: (i) harmonic damping from a white dwarf magnetosphere with constant density $n_{\text {th }}=10^{7} \mathrm{~cm}^{-3}$, and thermal temperature $k_{\mathrm{B}} T_{\mathrm{th}}=1 \mathrm{eV}$; (ii) $1 \%$ of current electrons in the unipolar inductor circuit contribute to the mildly relativistic loss-cone electron distribution, with mean energy of $1 \mathrm{keV}$ (provided that the induced potential across the planet exceeds $1 \mathrm{kV}$ ); (iii) an angular loss-cone width $\Delta \alpha=0.01$; and (iv) a source-observer distance of $100 \mathrm{pc}$.

Figure 7 displays the predicted flux densities at the observing frequencies 5, 8, 43, 100, 250 and $650 \mathrm{GHz}$. Points to note concerning Fig. 7 are that: (i) Greater than micro-Jansky flux densities are predicted over a relatively broad range of $\mu-P$ space (where radio detections are most likely in the grey 
detection band); (ii) The predicted flux densities are strongly frequency dependent; (iii) The predicted radio emissions are $100 \%$ circularly polarized, with the two humps in Figs. 7c-f corresponding to $o$-mode polarization at low $P$ and $x$-mode polarization at high $P$. At $5 \mathrm{GHz}$ and $8 \mathrm{GHz}$, (Figs. $7 \mathrm{a}$ and b), only $o$-mode emission is predicted. Predominantly $x$-mode polarization is predicted for $43,100,250$ and $650 \mathrm{GHz}$, because the $o$-mode hump lies to the left of the detection band (in the short system lifetime regime). Therefore a reversal in the sense of circular polarization is predicted at a frequency between 8 and $43 \mathrm{GHz}$ (although it should be noted that this transition frequency is strongly dependent on the choice of model free parameters). (iv) Radio flux densities exceeding $1 \mathrm{Jy}$ are predicted at observing frequencies of 250 and $650 \mathrm{GHz}$. However these values occur towards the left-hand edge of the detection band, and are correlated with shorter unipolar inductor lifetimes $\left(\sim 10^{7}-10^{8} \mathrm{yr}\right)$, and are therefore less likely to be observed than systems with lower predicted flux densities towards the right-hand side of the detection band. (v) By analogy to the Jupiter-Io radio emissions, the predicted radio emission will have short duty cycles (of typically a few percent), with one or two pulses recurring at the orbital period. The predicted inter-pulse period ranges from $\sim 0.1-\sim 100$ days (see Fig. 2) and therefore long observing times are required.

The lowest observing frequency displayed in Fig. $7(5 \mathrm{GHz})$ is close to operating frequencies of the VLA, ATCA, Merlin, Effelsberg and Arecibo radio telescopes. The frequencies $8 \mathrm{GHz}$ and $43 \mathrm{GHz}$ also lie within VLA operating bands. The highest chosen frequencies in Fig. 7 (100, 250, and $650 \mathrm{GHz})$ relate to ALMA operating frequencies. We assume sensitivity limits for the VLA and ALMA below, based on an observing time of one hour, and taking into account the ( 7-10 times) reduction in signal-to-noise associated with an assumed 1-2\% duty cycle of the beamed radio pulses. The VLA can achieve a point-source rms sensitivity of $50 \mu \mathrm{Jy}$ at $8 \mathrm{GHz}$ (with a similar value for $5 \mathrm{GHz}$ ), and $0.25 \mathrm{mJy}$ at $43 \mathrm{GHz}$ in ten minutes. This corresponds to a detection limit for the VLA of $0.1 \mathrm{mJy}$ at 5 and $8 \mathrm{GHz}$, and $1 \mathrm{mJy}$ at $43 \mathrm{GHz}$. This detection limit can be lowered further by compensating for the degradation of the signalto-noise ratio over a full orbital period, by using data-folding or power spectrum analytical techniques. Similarly, ALMA can achieve a sensitivity of $\sim 0.01 \mathrm{mJy}$ at $100 \mathrm{GHz}, \sim 0.04 \mathrm{mJy}$ at $250 \mathrm{GHz}$, and $\sim 0.3 \mathrm{mJy}$ at $650 \mathrm{GHz}$ in ten minutes. This corresponds to a detection limit of $0.1 \mathrm{mJy}$ at 100 and $250 \mathrm{GHz}$, and $1 \mathrm{mJy}$ at $650 \mathrm{GHz}$.

The predicted peak flux densities at 5 and $8 \mathrm{GHz}$ (Figs. 7a and b) lie below $10 \mu \mathrm{Jy}$ and are therefore unlikely to be detected with the VLA or other existing radio telescopes at these frequencies. Detection of white dwarf/planet systems, if they exist, at frequencies below $20 \mathrm{GHz}$ is more likely with nextgeneration radio telescopes such as the Square Kilometre Array (with a proposed maximum frequency of $\sim 20 \mathrm{GHz}$ ), which will achieve sensitivities below $1 \mu \mathrm{Jy}$.

The predicted peak flux densities at $43 \mathrm{GHz}$ are of the same order as the VLA detection limit at this frequency $(1 \mathrm{mJy})$. Thus detections at $43 \mathrm{GHz}$ using the VLA are most probable for observation times well exceeding one hour, and/or if the model assumptions presented in Fig. 7c are too conservative.
Based on the radio emission model developed by Willes $\& \mathrm{Wu}$ (2004), detection of white dwarf/planet systems appears most likely at frequencies exceeding $\sim 80 \mathrm{GHz}$. For ALMA, the optimum observing frequencies are at $100 \mathrm{GHz}$ and $250 \mathrm{GHz}$, where the predicted flux densities (within the detection band) exceed $0.1 \mathrm{mJy}$ over a significant area in the $\mu-P$ plane. Higher fluxes are predicted at $650 \mathrm{GHz}$ than at $250 \mathrm{GHz}$; however, only the tail of the $x$-mode peak lies within the detection band, with a smaller area of the $\mu-P$ plane exceeding (the higher detection limit of) $1 \mathrm{mJy}$. The likelihood of detection diminishes further at higher frequencies because the predicted flux density peaks lie to the left of the detection band, and thus the white dwarf/planet systems are too short-lived to be likely to be detected.

\section{The white-dwarf/planet radio luminosity function}

We now compute the radio luminosity function for whitedwarf/planet systems, based on the model discussed in the previous section. This allows us to predict the number of detectable systems for various observational settings. We use a Monte Carlo algorithm and consider a volume-limited sample. The following assumptions are made in the simulation: (i) All systems are within a sphere with a radius of $1 \mathrm{kpc}$ centred at the observer. The spatial distribution of the systems is such that the probability to find one at any point in the sphere is equal. We assume a local white dwarf number density of $3.4 \times 10^{-3} \mathrm{pc}^{-3}$ (Leggett et al. 1998), and and assume that $\sim 10 \%$ of white dwarfs are magnetized (Wickramasinghe \& Ferrario 2000; Gaensicke et al. 2002; Liebert et al. 2003), Thus, the total number of magnetized white dwarfs within the 1-kpc sphere is $1.4 \times 10^{6}$. Note that we slightly underestimate the number of detectable systems because we do not count radio-bright systems beyond $1 \mathrm{kpc}$. (ii) The radiation beaming pattern (on the surface of a hollow cone, centred on the active magnetic flux tube in both hemispheres, with opening angle $>60^{\circ}$ and beam width of several degrees) intersects the source-observer line at least once per orbital period. This is a reasonable assumption as it is consistent with the beaming geometry of a nearby unipolar-inductor system, the Jupiter-Io system. In this simulation, we assume that the orientations of the systems are not randomly distributed, and that the magnetic and orbital rotation axes are nearly aligned in each system. Relaxing these assumptions should not dramatically affect these predictions. (iii) The white-dwarf masses have a normal distribution, with a mean of $0.562 M_{\odot}$ and a standard deviation of 0.1 . These values are similar to those of an observational sample of 1175 DA white dwarfs of (Madej et al. 2004). We use the Hamada \& Salpeter (1961) relation to derive the radii of the white dwarfs in our simulated sample. (iv) The distribution of white dwarf magnetic moments is modelled with a log-normal distribution, with a mean $\left\langle\log _{10}\left[\mu\left(\mathrm{G} \mathrm{cm}^{-3}\right)\right]\right\rangle=22$ and a standard deviation of 1 . This approximates the distribution of measured magnetic white dwarf moments (Wickramasinghe \& Ferrario 2000, Fig. 23). (v) The orbital period is assumed to be evenly distributed in the range $3<\log _{10} P<8$ (corresponding to orbital separations from near zero to $\sim 1.5 \mathrm{AU}$ ). (vi) Only white dwarf/planet 


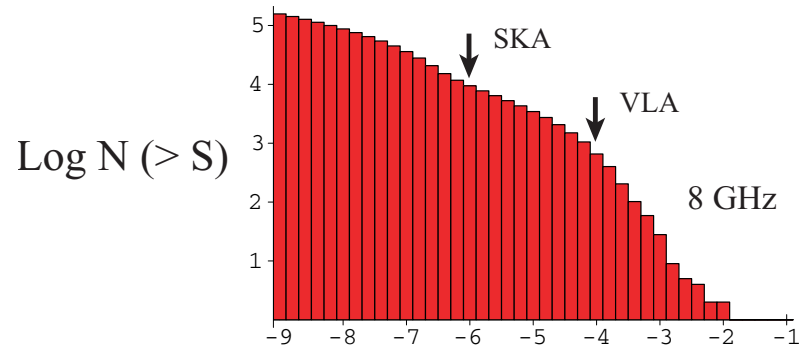

$\log \mathrm{S} \quad(\mathrm{Jy})$

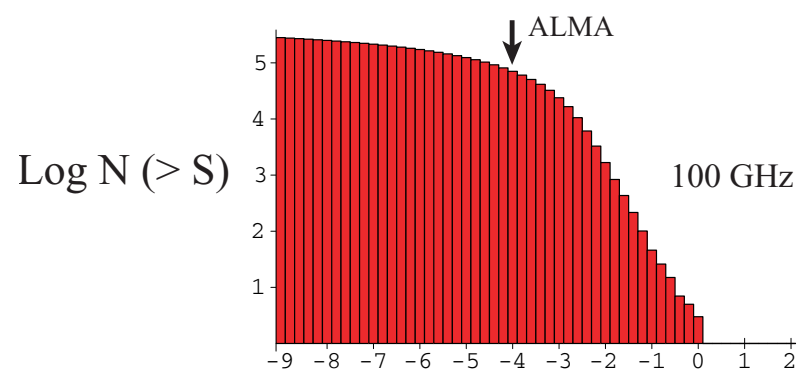

$\log \mathrm{S}(\mathrm{Jy})$

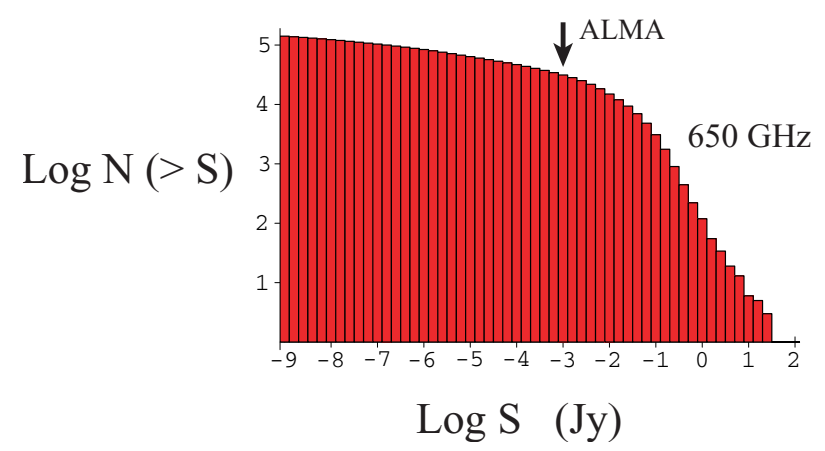

Fig. 8. Predicted radio luminosity functions (number of systems $N$ with flux densities at the observer exceeding $S$ ) from a Monte-Carlo simulation of a volume-limited sample of white-dwarf/planet systems at a) $8 \mathrm{GHz}$, b) $100 \mathrm{GHz}$, and c) $650 \mathrm{GHz}$.

systems with magnetic moments and orbital periods which lie inside the detection band shown in Fig. 2 are considered to be detectable.

Figure 8 displays the cumulative radio luminosity functions (number of sources $N$ exceeding a specified radio flux density $S$ ) at (a) $8 \mathrm{GHz}$, (b) $100 \mathrm{GHz}$, and (c) $650 \mathrm{GHz}$. The luminosity functions are unnormalized such that they contain the implicit assumption that each magnetic white dwarf hosts a terrestrial planet. As discussed in Sect. 3, the fraction of magnetic white dwarfs with terrestrial planets should be lower. The luminosity functions in Fig. 8 can be readily rescaled by multiplying the number of sources $N$ by the estimated fraction of magnetic white dwarfs with orbiting terrestrial planets (e.g. by $\Phi \sim 10^{-3}$, from Sect. 3).

The detection limits of the VLA, the SKA, and ALMA (see Sect. 4) are indicated by arrows in Fig. 8. We assume a detection limit of $1 \mu \mathrm{Jy}$ for the SKA at $8 \mathrm{GHz}$. Assuming a normalization factor of $10^{-3}$, less than one system is likely to be detected at $8 \mathrm{GHz}$ by the VLA. Approximately 10 systems will be detectable by the SKA at $8 \mathrm{GHz}$. This number will be higher if the SKA sensitivity at this frequency is lower than $1 \mu \mathrm{Jy}$ (by approximately an order of magnitude for an order of magnitude improvement in sensitivity). The number of detectable systems using the SKA will also increase with increasing observing frequency. For example, at $20 \mathrm{GHz}$ (the maximum SKA operating frequency for current SKA science requirements), the number of observable systems doubles to 20 . At $43 \mathrm{GHz}, \sim 5$ systems are likely to be detected by the VLA (not shown in Fig. 8). This will improve by a factor of 10 (to $\sim 50$ systems) with the order of magnitude increase in sensitivity of the planned Expanded VLA project (EVLA). At $100 \mathrm{GHz}, \sim 100$ systems will be detectable using ALMA. This increases to 200 systems at $250 \mathrm{GHz}$ (not shown), and decreases to $\sim 50$ systems at $650 \mathrm{GHz}$.

\section{Discussion and conclusions}

Since the discovery of the first planet around another star in 1995 , over 100 exoplanets have been found. Sensitivity limitations of optical telescopes have restricted discoveries to large (>Neptune-sized) planets. If these planets possess magnetic fields then they may also be detectable radio sources. However, the 5-50 parsec distance of known exoplanets places these potential radio sources at the margins of detectability with existing radio telescopes (Farrell et al. 1999) and radio searches to date have been unsuccessful (Bastian et al. 2000). Higher radio fluxes are expected from extrasolar Jupiter/Io-like systems, although for many known exoplanets the lifetimes of larger moons are limited by dynamic instabilities (Barnes \& O'Brien 2002). White-dwarf/terrestrial-planet systems, if they exist, are significantly stronger radio sources, due to the high white dwarf magnetic moments and the large planetary radius (relative to Jupiter and Io).

In this paper we make an estimate of the potential number of detectable white-dwarf/terrestrial-planet systems in our local stellar environment. We make the following findings:

(i) Terrestrial planets orbiting white dwarfs are only likely to be detected at radio wavelengths within a defined region in parameter space (i.e., the detection band in Fig. 2, as a function of white dwarf magnetic moment and orbital period). Within this parameter regime, sufficient power is generated in the unipolar inductor circuit to accelerate $\mathrm{keV}$ electrons for maser emission, and the system lifetime, which is limited by electrical dissipation, is not too short.

(ii) White-dwarf/terrestrial-planet systems can evolve from main sequence planetary systems, where the terrestrial planets not only survive the post-main-sequence stellar expansion phases, but can be dragged back into a closer orbit around the proto-white dwarf through a combination of tidal and engulfment drag effects. We estimate that $\approx 0.1 \%$ of main sequence planetary systems evolve into white-dwarf/terrestrial-planet systems capable of generating radio emissions. This number is most sensitive to variations in the initial stellar mass and mass-loss rate. Alternative evolutionary scenarios may also be 
responsible for the creation of close whitedwarf/terrestrial-planet systems. For instance, planets can attain closer orbits through interactions with other planets orbiting the same post-main-sequence star. Planets in stable orbits around a star undergoing rapid mass loss can become unstable to perturbations associated with interactions with adjacent planets, where the stability of each planetary orbit depends on the ratio of the planet mass to the stellar mass (Debes \& Sigurdsson 2002). Debes \& Sigurdsson (2002) suggested that planets may be observed in close orbits around white dwarfs if two or more planets become unstable to close approaches after the AGB phase and the ensuing interaction propels one planet into a closer orbit. Such a disruption of stable planetary orbits is more likely for larger (Jupiter-sized) planets, but may also be important for surviving terrestrial planets. Another potential source of close-orbit "terrestrial" planets is the remnant solid cores of gas giant planets which escape permanent engulfment after the evaporation of the outer gas layers during temporary engulfments in the late AGB/thermal pulse phases of stellar evolution.

(iii) We perform Monte-Carlo simulations based on observations of the local magnetic white dwarf number density, and the white dwarf mass and magnetic moment distributions, combined with the Willes \& Wu (2004) radio flux density model. This allows the prediction of the number of detectable white-dwarf/planet systems as a function of telescope sensitivity. We find that the optimum frequency range for detecting these systems is from $\sim 50$ to $\sim 500 \mathrm{GHz}$. For instance, between 10 and 100 detectable systems are anticipated in each of the ALMA observing bands around 100, 250 and $650 \mathrm{GHz}$, and $\sim 50$ systems from the Expanded VLA (EVLA) at $43 \mathrm{GHz}$. It appears unlikely that existing radio telescopes will detect radio emissions from white-dwarf/planet systems at or below $43 \mathrm{GHz}$. The higher sensitivity of the planned SKA will increase the likelihood of detection at or below $20 \mathrm{GHz}$ (with $\sim 20$ detectable systems at $20 \mathrm{GHz}$, based on an assumed sensitivity of $1 \mu \mathrm{Jy}$ ). We stress that these estimates of the number of detectable systems are dependent on the free parameters in the radio flux model, and the estimated fraction of magnetic white dwarfs which host terrestrial planets in close orbits.

Acknowledgements. The authors thank the referee for advice which substantially improved this paper. A.J.W. acknowledges support from the Australian Research Council as an Australian Postdoctoral Fellow, and thanks the British Antarctic Survey (Cambridge) for their hospitality during a research sabbatical.

\section{References}

Barnes, J. W., \& O'Brien, D. P. 2002, ApJ, 575, 1087

Bastian, T. S., Dulk, G. A., \& Leblanc, Y. 2000, ApJ, 545, 1058

Bigg, E. K. 1964, Nature, 203, 1008

Boothroyd, A. I., \& Sackmann, I.-J. 1988a, ApJ, 328, 632

Boothroyd, A. I., \& Sackmann, I.-J. 1988b, ApJ, 328, 653

Chanmugam, G., \& Dulk, G. A. 1983, Annals of Israel Phys. Soc., 19, 223

Clarke, J. T., Ajello, J., Ballester, G., et al. 2002, Nature, 415, 997

Debes, J. H., \& Sigurdsson, S. 2002, ApJ, 572, 556

Delamere, P. A., Bagenal, F., Ergun, R., Su, Y.-J., et al. 2003, J. Geophys. Res., 108, 1241

Dulk, G. A. 1967, Icarus, 7, 173

Farrell, W. M., Desch, M. D., \& Zarka, P. 1999, J. Geophys. Res., 104, 14025

Gaensicke, B. T., Euchner, F., \& Jordan, S. 2002, A\&A, 394, 957

Goldreich, P., \& Lynden-Bell, D. 1969, ApJ, 156, 59

Hamada, T., \& Salpeter, E. 1961, ApJ, 134, 683

Hakala, P., Ramsay, G., Wu, K., et al. 2003, MNRAS, 343, L10

Hewitt, R. G., Melrose, D. B., \& Rönnmark, K. G. 1981, PASA, 4, 221

Hill, T. W., \& Vasyliunas, V. M. 2002, J. Geophys. Res., 107, 1464

Jørgensen, U. G. 1991, A\&A, 246, 118

Kaiser, M. L., et al. 2000, J. Geophys. Res., 105, 16053

Leggett, S. K., Ruiz, M. T., \& Bergeron, P. 1998, ApJ, 497, 294

Li, J., Ferrario, L., \& Wickramasinghe, D. T. 1998, ApJ, 503, L151

Liebert, J., Bergeron, P., \& Holberg, J. B. 1998, AJ, 125, 348

Madej, J., Nalezyty, M., \& Althaus, L. G. 2004, A\&A, 419, L5

Maeda, K., \& Carr, T. D. 1992, J. Geophys. Res., 97, 1549

Mandell, A. M., \& Sigurdsson, S. 2003, ApJ, 599, L111

Melrose, D. B., \& Dulk, G. A. 1982, ApJ, 259, 844

Menietti, J. D., Gurnettt, D. A., Kurth, W. S., \& Groene, J. B. 1998, Geophys. Res. Lett., 25, 4281

Menietti, J. D., Gurnettt, D. A., \& Christopher, I. 2001, Geophys. Res. Lett., 28, 3047

Piddington, J. H., \& Drake, J. F. 1968, Nature, 217, 935

Ramsay, G., Wu, K., Cropper, M., et al. 2002, MNRAS, 333, 575

Rasio, F. A., Tout, C. A., Lubow, H., \& Livio, M. 1996, ApJ, 470, 1187

Retter, A., \& Maron, A. 2003, MNRAS, 345, L25

Rybicki, K. R., \& Denis, C. 2001, Icarus, 151, 130

Sackmann, I.-J., Boothroyd, A. I., \& Kraemer, K. E. 1993, ApJ, 418, 457

Soker, N. 2001, MNRAS, 324, 699

Soker, N., \& Livio, M. 1994, ApJ, 421, 219

Strohmayer, T. E. 2002, ApJ, 581, 577

Strohmayer, T. E. 2003, ApJ, 593, L39

Verbunt, F., \& Phinney, E. S. 1995, A\&A, 296, 709

Wetherill, G. W. 1996, Icarus, 119, 219

Wickramasinghe, D. T., \& Ferrario, L. 2000, PASP, 112, 873

Willes, A. J., \& Wu, K. 2004, MNRAS, 348, 285

Wu, K., Cropper, M., Ramsay, G., Sekiguchi, K., et al. 2002, MNRAS, 331,221 